\title{
Dimensions of Invariant Sets of Expanding Maps
}

\author{
Huyi $\mathrm{Hu}^{\star}$ \\ Department of Mathematics, University of Maryland, College Park, MD 20742, USA
}

Received: 2 February 1994

\begin{abstract}
We consider a compact invariant set $\Lambda$ of an expanding map of a manifold $M$ and give upper and lower bounds for the Hausdorff Dimension $\operatorname{dim}_{H}(\Lambda)$, and box dimensions $\operatorname{dim}_{B}(\Lambda)$ and $\overline{\operatorname{dim}}_{B}(\Lambda)$. These bounds are given in terms of the topological entropy, topological pressure, and uniform Lyapunov exponents of the map.
\end{abstract}

A measure-theoretic version of these results is also included.

\section{Introduction}

It is well known that many self-similar sets, or fractals, can be realized as invariant sets of smooth expanding maps. The purpose of this paper is to give estimates on the box dimensions and Hausdorff dimensions of these sets.

Most of the known results on dimensions in dynamical systems are of the following types. Some are about the dimensions of invariant measures (see e.g. [L] and [LY]). Some are about the dimensions of sets constructed via a sequence of affine contractions (e.g. [F1] and [GL]). Others deal with subsets of the line. See also [G, MM, BU], etc.

At present, there are few known effective ways to calculate the box and Hausdorff dimensions of sets (sets, not measures) invariant under nonlinear maps in dimensions larger than 1 . In this paper we consider invariant sets of uniformly expanding maps. We give upper and lower bounds for box and Hausdorff dimensions in terms of topological entropy, topological pressure, and uniform Lyapunov exponents. Examples of self-affine sets show that these bounds are sharp (see Sect. 1, Example 2). We will also give parallel results for dimensions of invariant measures.

We state our results.

Let $M$ be an $m$-dimensional $C^{\infty}$ Riemannian manifold with Riemannian measure $v$, and $f$ be a $C^{2}$ map from $M$ to itself. Let $\Lambda \subset M$ be an $f$-invariant compact subset, where $f$-invariance means $f \Lambda=\Lambda$. We also assume that $f$ is expanding

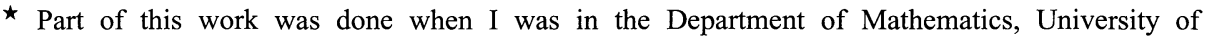
Arizona.
} 
on $\Lambda$, i.e., there exist $C>0, \kappa>1$, such that $\forall x \in \Lambda, n \geqq 1$,

$$
\left\|D f^{n}(x) u\right\| \geqq C \kappa^{n}\|u\| \quad \forall u \in T_{x} M .
$$

Without loss generality we can take $C=1$.

We denote by $|D f(x)|$ the Jacobian of $D f(x): T_{x} M \rightarrow T_{f x} M$, by $\|D f(x)\|$ the norm of $D f(x)$ and by $\llbracket D f(x) \rrbracket$ the minimum norm of $D f(x)$, i.e., $\| D f(x) \rrbracket=$ $\inf _{0 \neq u \in T_{x} M} \frac{\|D f(x) u\|}{\|u\|}$. Put

$$
\sigma_{+}=\lim _{n \rightarrow \infty} \frac{1}{n} \log \left\|\left.D f^{n}\right|_{\Lambda}\right\| \quad \text { and } \quad \sigma_{-}=\lim _{n \rightarrow \infty} \frac{1}{n} \log \|\left. D f^{n}\right|_{\Lambda} \rrbracket,
$$

where $\left\|\left.D f^{n}\right|_{\Lambda}\right\|=\sup _{x \in \Lambda}\left\|D f^{n}(x)\right\|$ and $\left\|\left.D f^{n}\right|_{\Lambda}\right\|=\inf _{x \in \Lambda}\left\|D f^{n}(x)\right\|$. We call $\sigma_{+}$ and $\sigma_{-}$the largest and smallest uniform Lyapunov exponents respectively. Since $f$ is expanding on $\Lambda, \sigma_{-}>0$.

We also denote $h_{\text {top }}=h\left(\left.f\right|_{\Lambda}\right)$, the topological entropy, and $P=P\left(\left.f\right|_{\Lambda},-\log \right.$ $|D f(x)|)$, the topological pressure of $\left.f\right|_{\Lambda}$ for the function $-\log |D f(x)|$. The Hausdorff dimension, lower and upper box dimensions of $\Lambda$ are denoted by $\operatorname{dim}_{H}(\Lambda), \underline{\operatorname{dim}}_{B}(\Lambda)$ and $\overline{\operatorname{dim}}_{B}(\Lambda)$ respectively. Their precise definitions are given in Sect. 2.

Theorem A. Let $f$ be a $C^{2}$ self-map of an m-dimensional Riemannian manifold $M$, and let $\Lambda$ be a compact $f$-invariant set. Assume that $f$ is expanding on $\Lambda$. Then

i) $\frac{h_{\text {top }}}{\sigma_{+}} \leqq D(\Lambda) \leqq \frac{h_{\text {top }}}{\sigma_{-}}$, and

ii) $m+\frac{P}{\sigma_{-}} \leqq D(\Lambda) \leqq m+\frac{P}{\sigma_{+}}$,

where $D(\Lambda)$ is $\operatorname{dim}_{H}(\Lambda), \underline{\operatorname{dim}}_{B}(\Lambda)$, or $\overline{\operatorname{dim}}_{B}(\Lambda)$.

To prove the lower bound for $D(\Lambda)$, we use part of the following theorem, which is a measure-theoretic version of Theorem A.

For an ergodic measure $\mu$ on $\Lambda$, we let $h=h_{\mu}(f)$ denote the measure-theoretic entropy, $\lambda_{1}$ and $\lambda_{s}$ be the largest and smallest Lyapunov exponents, and $\lambda$ be the sum of all Lyapunov exponents including multiplicities - all of them depend on $\mu$. The definitions related to dimensions of measures are stated in Sect. 2.

Theorem B. Let $f$ be a $C^{2}$ self-map of an m-dimensional Riemannian manifold $M$, and let $\Lambda$ be a compact $f$-invariant set. Assume that $f$ is expanding on $\Lambda$ and $\mu$ is an ergodic probability measure on $\Lambda$. Then

i) $\frac{h}{\lambda_{1}} \leqq D(\mu) \leqq \frac{h}{\lambda_{s}}$, and

ii) $m-\frac{\lambda-h}{\lambda_{s}} \leqq D(\mu) \leqq m-\frac{\lambda-h}{\lambda_{1}}$,

where $D(\mu)$ is $\operatorname{dim}_{H}(\mu), \underline{\operatorname{dim}}_{B}(\mu), \overline{\operatorname{dim}}_{B}(\mu), \underline{\operatorname{dim}}_{L}(\mu)$, or $\overline{\operatorname{dim}}_{L}(\mu)$.

Remark. Theorem B is a generalization of a known result that if $f$ is conformal then the entropy $h_{\mu}(f)$ is equal to the product of the Lyapunov exponent and the Hausdorff dimension (see [R]). Also, it has been conjectured that the formula of Ledrappier and Young for $\operatorname{dim}_{H}(\mu)$ is true for invariant measures of smooth expanding maps. The results in Theorem B are consistent with this conjecture.

The major work in this paper is to prove part ii) of Theorem B. Proposition 2 in Sect. 4 plays a key role for the proof. 


\section{Examples}

We begin by looking at some examples.

Example 1. Let $R_{0}$ be a unit square in $\mathbb{R}^{2}$. Take real numbers $1<a<b$. Put $n$ rectangles $R_{1}, \ldots, R_{n}$ of width $a^{-1}$ and height $b^{-1}$ in an arbitrary way, as long as they are separated from each other. Define a $C^{2}$ map $f$ on $\mathbb{R}^{2}$ such that $f$ maps each of $R_{i}$ affinely onto $R_{0}$. The $n$ inverses of $\left.f\right|_{R_{i}}: R_{i} \rightarrow R_{0}, i=1, \ldots, n$, determine an $f$-invariant set, or fractal, $\Lambda \subset \bigcup_{i=1}^{n} R_{i}$ in a usual way (see [H or F2]). In fact, $\Lambda=\bigcap_{n=0}^{\infty} f^{-i}\left(R_{1} \cup \cdots \cup R_{n}\right)$, which is an invariant set of $f$.

Clearly, $f$ is expanding on $A$ with expanding rates $a$ and $b$ in horizontal and vertical directions, and the smaller and larger uniform Lyapunov exponents of $f$ are $\sigma_{-}=\log a$ and $\sigma_{+}=\log b$ respectively. It is also easy to see that $h_{\text {top }}=$ $h\left(\left.f\right|_{\Lambda}\right)=\log n$. Since $-\log |D f(x)|=-\log a-\log b$ is a constant on $\Lambda, P=$ $P\left(\left.f\right|_{\Lambda},-\log |D f(x)|\right)=h_{\text {top }}\left(\left.f\right|_{\Lambda}\right)-\log a-\log b=\log n-\log a-\log b$

(see [W] Sect. 9.2). By Theorem A we get that

$$
\max \left\{\frac{\log n}{\log b}, 1+\frac{\log n-\log b}{\log a}\right\} \leqq D(\Lambda) \leqq \min \left\{\frac{\log n}{\log a}, 1+\frac{\log n-\log a}{\log b}\right\} .
$$

In this case all the terms in the bounds are explicit.

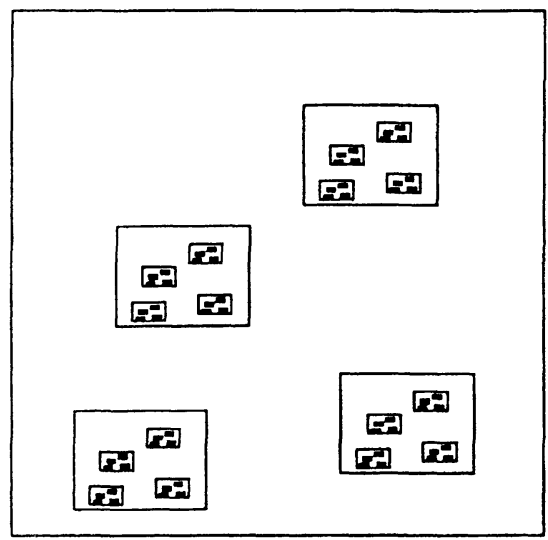

Fig 1.

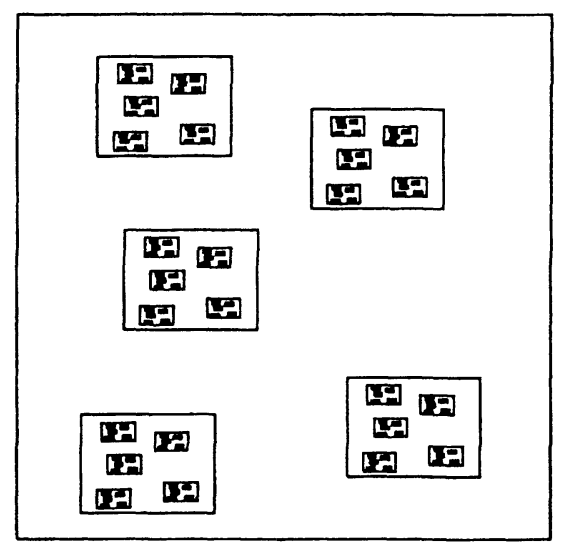

Fig 2.

In Fig. $1, a=4.1, b=4.9$ and $n=4$. Theorem A gives $0.982 \cdots \leqq D(\Lambda) \leqq$ $0.984 \cdots$, which is less than 1. In Fig. 2, we use the same $a$ and $b$, but $n=5$. By Theorem $\mathrm{A}$, we get $1.014 \cdots \leqq D(\Lambda) \leqq 1.140 \cdots$, which is larger than 1 . (In these figures the black rectangles form the set $\bigcap_{k=0}^{2} f^{-k}\left(\bigcup_{i=1}^{n} R_{i}\right)$.)

Example 2 (Generalized Sierpinski carpets). Let $R_{0}$ be a unit square in $\mathbb{R}^{2}$. Take integers $0<p<q$. Partition $R_{0}$ into $p$ columns and $q$ rows, producing $p q$ rectangles of size $p^{-1} \times q^{-1}$. Select a subcollection of these rectangles $\mathscr{R}=\left\{R_{1}, \ldots, R_{n}\right\}$, and generate an invariant set $\Lambda$ in the same way as in the last example. (If we identify $R_{0}$ with a torus, then $f$ is a $C^{2}$ map even though some of rectangles are adjacent.) The dimensions of the set $\Lambda$ were studied by McMullen ([Mc]) and Bedford ([B]). 
The box and Hausdorff dimensions of $\Lambda$ are

$$
\begin{aligned}
\operatorname{dim}_{B}(\Lambda) & =\frac{\log \hat{p}}{\log p}+\frac{1}{\log q} \cdot \log \left(\frac{1}{\hat{p}} \sum_{j=1}^{p} n_{j}\right) \\
\operatorname{dim}_{H}(\Lambda) & =\frac{1}{\log p} \log \left(\sum_{j=1}^{p}\left(n_{j}\right)^{\frac{\log p}{\log q}}\right)
\end{aligned}
$$

where $n_{j}$ denotes the number of rectangles selected from the $j^{\text {th }}$ column and $\hat{p}$ is the number of columns containing at least one rectangle in $\mathscr{R}$. (See also [F2].) These results are consistent with our estimates.

Note that in these examples the dimensions of $\Lambda$ depend not only on the number $n$ of selected rectangles but also on the configuration of the rectangles in $\mathscr{R}$. This shows that in general one cannot get an exact formula in terms of $\sigma_{-}, \sigma_{+}, h_{\text {top }}$ and $P$ for either box dimensions or Hausdorff dimensions.

In fact, by choosing suitable configurations of the rectangles, the bounds in Theorem A can be reached. Let us look at the following cases.

i) Take $n_{i}=0$ or 1 for all $1 \leqq i \leqq p$. Thus $n=\hat{p}$. By (1.2) we get that $\operatorname{dim}_{B}(\Lambda)=\operatorname{dim}_{H}(\Lambda)=\frac{\log \hat{p}}{\log p}$, which is one of the upper bounds in (1.1).

ii) For all $1 \leqq i \leqq p$, put $n_{i}=k$. In this case, $n=k p$. We obtain that $\operatorname{dim}_{B}(\Lambda)=\operatorname{dim}_{H}(\Lambda)=1+\frac{\log k}{\log q}$. This coincides the other upper bounds in (1.1).

iii) Choose $\hat{p}=1$, i.e. all selected rectangles are in one column. Hence only one $n_{i}$ is greater than 0 and $n$ is equal to this $n_{i}$. Equation (1.2) implies that $\operatorname{dim}_{B}(\Lambda)=\operatorname{dim}_{H}(\Lambda)=\frac{\log n}{\log p}$, one of the lower bounds in (1.1).

iv) Let $n_{i}$ be equal to either 0 or $q$. So $n=\hat{p} \cdot q$, and $\operatorname{dim}_{B}(\Lambda)=\operatorname{dim}_{H}(\Lambda)=$ $1+\frac{\log \hat{p}}{\log p}$, same as the other lower bound.

Example 3 (Similitudes). Let $\Lambda$ be constructed as in Example 2 but put $p=q>0$. In this case $f$ is called a similitude (see $[\mathrm{H}]$ ). Since $\sigma_{+}=\sigma_{-}=\log p$, the estimates in Theorem A become equality. Thus, we obtain that

$$
\operatorname{dim}_{H}(\Lambda)=\operatorname{dim}_{B}(\Lambda)=\frac{\log n}{\log p} .
$$

In general, for any invariant set $\Lambda$ of a similitude $f$,

$$
\operatorname{dim}_{H}(\Lambda)=\operatorname{dim}_{B}(\Lambda)=\frac{h_{\text {top }}}{\log \left\|\left.D f\right|_{\Lambda}\right\|} .
$$

\section{Definitions and Notations}

First we give the definitions of the various notions of dimensions. (See e.g. [F2].) For a metric space $X$, its Hausdorff dimension is defined by

$$
\operatorname{dim}_{H}(X)=\inf \left\{\alpha: \lim _{\varepsilon \rightarrow 0} \inf _{\operatorname{diam} \mathscr{C}<\varepsilon} \sum_{A \in \mathscr{C}}(\operatorname{diam} A)^{\alpha}=0\right\},
$$


where $\mathscr{C}$ is a cover of $X$ and $\operatorname{diam} \mathscr{C}=\inf \{\operatorname{diam} A: A \in \mathscr{C}\}$. If $v$ is a probability measure on $X$, then the Hausdorff dimension of the measure $v$ is given by

$$
\operatorname{dim}_{H}(v)=\inf \left\{\operatorname{dim}_{H}(Y): Y \subset X, v Y=1\right\} .
$$

The upper and lower box dimensions of $X$ are defined by

$$
\overline{\operatorname{dim}}_{B}(X)=\limsup _{\varepsilon \rightarrow 0} \frac{\log N(\varepsilon)}{-\log \varepsilon} \text { and } \underline{\operatorname{dim}}_{B}(X)=\liminf _{\varepsilon \rightarrow 0} \frac{\log N(\varepsilon)}{-\log \varepsilon}
$$

respectively, where $N(\varepsilon)$ denotes the minimum number of balls of radius $\varepsilon$ which cover $X . \overline{\operatorname{dim}}_{B}(X)$ and $\underline{\operatorname{dim}}_{B}(X)$ are also called upper and lower capacities. If $\overline{\operatorname{dim}}_{B}(X)=\underline{\operatorname{dim}}_{B}(X)$, then we simply call this number the box dimension, and denote it by $\operatorname{dim}_{B}(X)$. Similarly, we have

$$
\overline{\operatorname{dim}}_{B}(v)=\lim _{\delta \rightarrow 0} \inf \left\{\overline{\operatorname{dim}}_{B}(Y): Y \subset X, v Y>1-\delta\right\},
$$

and

$$
\underline{\operatorname{dim}}_{B}(v)=\lim _{\delta \rightarrow 0} \inf \left\{\underline{\operatorname{dim}}_{B}(Y): Y \subset X, v Y>1-\delta\right\} .
$$

F. Ledrappier has introduced the following notions [L]:

$$
\overline{\operatorname{dim}}_{L}(v)=\lim _{\delta \rightarrow 0} \limsup \frac{\log N(\varepsilon, \delta)}{-\log \varepsilon} \text { and } \underline{\operatorname{dim}}_{L}(v)=\lim _{\delta \rightarrow 0} \lim _{\varepsilon \rightarrow 0} \inf \frac{\log N(\varepsilon, \delta)}{-\log \varepsilon},
$$

where $N(\varepsilon, \delta)$ is the minimum number of balls of diameter $\varepsilon$ covering a subset in $X$ of measure greater than $1-\delta$.

The following inequalities are immediate:

$$
\operatorname{dim}_{H}(v) \leqq \operatorname{dim}_{H}(X),
$$

$$
\underline{\operatorname{dim}}_{L}(v) \leqq \underline{\operatorname{dim}}_{B}(v) \leqq \underline{\operatorname{dim}}_{B}(X), \quad \overline{\operatorname{dim}}_{L}(v) \leqq \overline{\operatorname{dim}}_{B}(v) \leqq \overline{\operatorname{dim}}_{B}(X),
$$

and

$$
\operatorname{dim}_{H}(X) \leqq \underline{\operatorname{dim}}_{B}(X) \leqq \overline{\operatorname{dim}}_{B}(X) .
$$

Moreover, L.-S. Young has proved ([Y]) that

$$
\operatorname{dim}_{H}(v) \leqq \underline{\operatorname{dim}}_{L}(v) .
$$

The local entropy of $f$ at $x$ with respect to $\mu$ is defined as (see [BK])

$$
\begin{aligned}
h_{\mu}(x, f) & =\lim _{\varepsilon \rightarrow 0} \limsup _{n \rightarrow \infty}-\frac{1}{n} \log \mu B_{n}(x, \varepsilon) \\
& =\lim _{\varepsilon \rightarrow 0} \liminf _{n \rightarrow \infty}-\frac{1}{n} \log \mu B_{n}(x, \varepsilon) .
\end{aligned}
$$

It satisfies $h_{\mu}(f x, f)=h_{\mu}(x, f)$ and $\int h_{\mu}(x, f) d \mu(x)=h_{\mu}(f)$, where $h_{\mu}(f)$ is the measure-theoretic entropy of $f$. Since $\mu$ is ergodic, we can write $h=h_{\mu}(x, f)$ $\mu$-a.e. $x$.

Let

$$
B(x, \alpha)=\{y \in M: d(y, x) \leqq \alpha\}
$$


and

$$
B_{n}(x, \alpha)=\left\{y \in M: d\left(f^{i} y, f^{i} x\right) \leqq \alpha, i=0, \ldots, n\right\} .
$$

$B(x, \alpha)$ and $B_{n}(x, \alpha)$ are called the $\alpha$-ball and $(n, \alpha)$-ball about $x$ respectively. If $\Gamma \subset M$, we denote

$$
B(\Gamma, \alpha)=\bigcup_{x \in \Gamma} B(x, \alpha), \quad B_{n}(\Gamma, \alpha)=\bigcup_{x \in \Gamma} B_{n}(x, \alpha) .
$$

\section{Preliminaries}

We denote by $\widehat{f}:(\widehat{M}, \widehat{\mu}) \rightarrow(\widehat{M}, \widehat{\mu})$ the inverse limit of $f:(M, \mu) \rightarrow(M, \mu)$. (For a precise definition, see e.g. [P] under the terminology "natural extension." Let $\pi_{i}: \widehat{M} \rightarrow M$ be the projection defined by $\pi_{i}\left(\left\{x_{j}\right\}_{j=0}^{\infty}\right)=x_{i} \forall\left\{x_{j}\right\}_{j=0}^{\infty} \in \widehat{M}$. For any $\widehat{x}=\left\{x_{i}\right\}, \widehat{f}\left\{x_{i}\right\}=\left\{f x_{i}\right\}$. The inverse of $\widehat{f}$ is given by $\widehat{f}^{-1}\left\{x_{i}\right\}=\left\{y_{i}\right\}$, where $y_{i}=x_{i+1}$.

Let

$$
\widehat{\Lambda}=\left\{\widehat{x}=\left\{x_{i}\right\} \in \widehat{M}: x_{i} \in \Lambda \forall i \geqq 0\right\} .
$$

Clearly, $\widehat{f} \widehat{\Lambda}=\widehat{\Lambda}$, and $\widehat{\mu}$ is an ergodic measure on $\widehat{\Lambda}$. It is also easy to see that with respect to cocycle $\left\{D f_{x_{0}}^{n}\right\}$, for $\widehat{\mu}$-a.e. $\widehat{x}=\left\{x_{i}\right\} \in \widehat{\Lambda}$, the Lyapunov exponents are identical to those in the system $f:(M, \mu) \rightarrow(M, \mu)$, i.e. $\lambda_{1}>\cdots>\lambda_{s}$.

Take $0<\gamma_{0} \leqq \frac{1}{200 m} \lambda_{s}$.

Let $\langle\langle\cdot, \cdot\rangle\rangle$ be the inner product on $T M$ given by the Riemannian structure which induces the norm $\|\cdot\|$. Let $\langle\cdot, \cdot\rangle$ and $|\cdot|$ denote the usual inner product and norm on $\mathbb{R}^{m}$ respectively. For $\alpha>0$, let $\tilde{B}(\alpha)$ be the ball in $\mathbb{R}^{m}$ centered at the origin of radius $\alpha$.

We now introduce the properties of Lyapunov charts $\left\{\Phi_{\hat{x}}: \widehat{x} \in \widehat{\Lambda}\right\}$ for $\widehat{f}$. The proof is similar with that in [LY].

Fact 1. $\forall \gamma \geqq 0$, there exists a measurable function $l: \widehat{\Lambda} \rightarrow[1, \infty]$ with $l\left(\widehat{f}^{ \pm 1} \widehat{x}\right) \leqq$ $l(\widehat{x}) e^{\gamma}$ and $l(\widehat{x})<\infty$ for $\mu$-a.e. $\widehat{x} \in \widehat{\Lambda}$, and a set of embeddings $\Phi_{\widehat{x}}: \tilde{B}\left(l(\widehat{x})^{-1}\right) \rightarrow$ $M$ at each point $\widehat{x} \in \widehat{\Lambda}$ such that the following holds.

i) $\Phi_{\hat{x}}(0)=x_{0}$, and the preimages $R_{i}(\widehat{x})=D \Phi_{\hat{x}}(0)^{-1}\left(E_{i}(\widehat{x})\right)$ of $E_{i}(\widehat{x})$ are $m u$ tually orthogonal in $\mathbb{R}^{m}$, where $i=1, \ldots, s$.

ii) Let $F_{\widehat{x}}=\Phi_{\widehat{f} \hat{x}}^{-1} \circ f \circ \Phi_{\widehat{x}}$ be the connecting map between the chart at $\hat{x}$ and the chart at $\widehat{f} \widehat{x}$. Then $\forall 1 \leqq i \leqq s, u \in R_{i}(\widehat{x}),|u| e^{\lambda_{i}-\gamma} \leqq\left|D F_{\hat{x}}(0) u\right| \leqq|u| e^{\lambda_{i}+\gamma}$.

iii) If $L(g)$ denotes the Lipschitz constant of the function $g$, then $L\left(F_{\hat{x}}^{ \pm}-\right.$ $\left.D F_{\widehat{x}}^{ \pm}(0)\right) \leqq \gamma$, and $L\left(D F_{\widehat{x}}^{ \pm}\right) \leqq l(\widehat{x})$.

iv) For all $v, v^{\prime} \in \tilde{B}\left(l(\widehat{x})^{-1}\right), K^{-1} d\left(\Phi_{\widehat{x}} v, \Phi_{\widehat{x}} v^{\prime}\right) \leqq\left|v-v^{\prime}\right| \leqq l(\widehat{x}) d\left(\Phi_{\widehat{x}} v, \Phi_{\widehat{x}} v^{\prime}\right)$ for some universal constant $K \geqq 1$.

We define $l: \Lambda \rightarrow[1, \infty]$ by $l(x)=\inf \left\{l(\widehat{x}): \widehat{x} \in \pi_{0}^{-1}(x)\right\}$. There is no ambiguity between $l(x)$ and $l(\widehat{x})$, because the domains are different. Clearly, $l(f x)$ $\leqq l(x) e^{\gamma}$.

For any $l \geqq 1$ let

$$
\Lambda_{l}=\{x \in \Lambda: l(x)<l\}
$$


Proposition 1. If $x \in \Lambda_{l}$ for some $l \geqq 1$, then $\forall n>0$,

$$
B\left(x, \varepsilon e^{-n\left(\lambda_{1}+2 \gamma\right)}\right) \subset B_{n}(x, \varepsilon K l) \quad \text { and } \quad B_{n}(x, \varepsilon) \subset B\left(x, \varepsilon K l e^{-n\left(\lambda_{s}-3 \gamma\right)}\right),
$$

provided $0<\varepsilon \leqq \frac{1}{2} l^{-2} e^{-2 n \gamma}$.

Proof. Take $z \in B\left(x, \varepsilon e^{-n\left(\lambda_{1}+2 \gamma\right)}\right)$.

Since $l(x)<l$, we can choose $\widehat{x} \in \pi_{0}^{-1}(x)$ such that $l \geqq l(\widehat{x})$. Denote $v=\Phi_{\widehat{x}}^{-1} z$, then by Fact 1. iv),

$$
|v| \leqq l(\widehat{x}) d(x, z) \leqq l(\widehat{x}) \cdot \varepsilon e^{-n\left(\lambda_{1}+2 \gamma\right)} \leqq \varepsilon l e^{-n\left(\lambda_{1}+2 \gamma\right)} .
$$

Write $F_{\widehat{x}}^{(n)}=F_{\widehat{f}}{ }^{n} \widehat{x} \circ \cdots \circ F_{\widehat{f} \widehat{x}} \circ F_{\widehat{x}}$. By Fact 1. ii) and iii) we have

$$
\left|F_{\widehat{x}}^{(n)} v\right| \leqq e^{n\left(\lambda_{1}+2 \gamma\right)}|v| \leqq e^{n\left(\lambda_{1}+2 \gamma\right)} \cdot \varepsilon l e^{-n\left(\lambda_{1}+2 \gamma\right)}=\varepsilon l \leqq \frac{1}{2} l(\widehat{x})^{-1} e^{-2 n \gamma} \leqq l\left(\widehat{f}^{n} \widehat{x}\right)^{-1}
$$

So $F_{\widehat{x}}^{(n)} v \in \tilde{B}\left(l\left(\widehat{f}^{n} \widehat{x}\right)^{-1}\right)$. Again, by Fact 1 . iv $)$,

$$
d\left(f^{n} x, f^{n} z\right) \leqq K\left|F_{\widehat{x}}^{(n)} v\right| \leqq K \cdot \varepsilon l=\varepsilon K l .
$$

It means $z \in B_{n}(x, \varepsilon K l)$.

The second inclusion can be proved similarly.

Recall that $\Lambda$ is a compact subset in an $m$-dimensional Riemannian manifold $M$ and $v$ is the Riemannian measure.

Lemma 1. $\exists K_{1} \geqq 1, \varepsilon_{1}>0$, such that $\forall 0<\varepsilon<\varepsilon_{1}, x \in \Lambda$,

$$
K_{1}^{-1} \varepsilon^{m} \leqq \nu B(x, \varepsilon) \leqq K_{1} \varepsilon^{m} .
$$

Proof. This is clear.

Lemma 2. $\exists K_{2} \geqq 1, \varepsilon_{2}>0$, such that $\forall 0<\varepsilon<\varepsilon_{2}, x \in \Lambda, n \geqq 0$,

$$
K_{2}^{-1} \varepsilon^{m} \leqq v B_{n}(x, \varepsilon)\left|D f^{n}(x)\right| \leqq K_{2} \varepsilon^{m} .
$$

Proof. Use distortion estimates $J^{-1} \leqq \frac{\left|D f^{n}(x)\right|}{\left|D f^{n}(y)\right|} \leqq J \forall y \in B_{n}(x, \varepsilon)$ and Lemma 1 .

Lemma 3. $\forall a>0$,

$$
\limsup _{n \rightarrow \infty}-\frac{1}{n a} \log \mu B\left(x, e^{-n a}\right) \quad \text { and } \quad \liminf _{n \rightarrow \infty}-\frac{1}{n a} \log \mu B\left(x, e^{-n a}\right),
$$

as functions of $x$, are constant for $\mu$-almost every $x$ in $\Lambda$.

Proof. There exists $k>0$ such that $\forall x \in A, f B\left(x, e^{-a n}\right) \subset B\left(f x, e^{-a(n-k)}\right)$ for all large $n$. So $\mu B\left(x, e^{-a n}\right) \leqq \mu B\left(f x, e^{-a(n-k)}\right)$. This implies both limits are $f$-invariant and therefore are constant almost everywhere.

Lemma 4. $\exists \varepsilon_{3}>0$, such that $\forall 0<\varepsilon<\varepsilon_{3} \mu$-a.e. $x \in \Lambda$,

$$
h=\lim _{n \rightarrow \infty}-\frac{1}{n} \log \mu B_{n}(x, \varepsilon) .
$$


Proof. Note that $f$ is expanding on $\Lambda$. If $\varepsilon_{3}$ is sufficiently small, then $\exists k>0$ such that for any $0<\varepsilon^{\prime} \leqq \varepsilon \leqq \varepsilon_{3}, B_{k}(x, \varepsilon) \subset B\left(x, \varepsilon^{\prime}\right) \subset B(x, \varepsilon) \forall x \in \Lambda$. Therefore, for any $n>0, B_{n+k}(x, \varepsilon) \subset B_{n}\left(x, \varepsilon^{\prime}\right) \subset B_{n}(x, \varepsilon) \forall x \in \Lambda$. Hence, we have

$$
\limsup _{n \rightarrow \infty}-\frac{1}{n} \log \mu B_{n}(x, \varepsilon)=\limsup _{n \rightarrow \infty}-\frac{1}{n} \log \mu B_{n}\left(x, \varepsilon^{\prime}\right) \quad \forall x \in \Lambda .
$$

This means that the above limit is independent of $\varepsilon$ provided $0<\varepsilon<\varepsilon_{3}$. So, by (2.5) $h=\lim \sup _{n \rightarrow \infty}-\frac{1}{n} \log \mu B_{n}(x, \varepsilon) \mu$-a.e. $x \in \Lambda$.

Similarly, we also have that $h=\liminf _{n \rightarrow \infty}-\frac{1}{n} \log \mu B_{n}(x, \varepsilon) \mu$-a.e. $x \in \Lambda$. The result then follows.

\section{Proof of Theorem B}

Proposition 2 is essential for the proof of the second part of Theorem B. It says that, up to factors $e^{-n \gamma}$, the measure of an $\varepsilon e^{-n \lambda_{s}}$-ball is greater than $e^{-n\left(m \lambda_{s}-\lambda+h\right)}$, and the measure of an $\varepsilon e^{-n \lambda_{1}}$-ball is less than $e^{-n\left(m \lambda_{1}-\lambda+h\right)}$. The idea of the proof comes from the following observation.

The volumes (i.e. the $v$-measure) of each $\varepsilon e^{-n \lambda_{s}}$-ball and each $(n, \varepsilon)$-ball are roughly $e^{-n m \lambda_{s}}$ and $e^{-n \lambda}$ respectively. Therefore an $\varepsilon e^{-n \lambda_{s}}$-ball can be covered by $\varepsilon e^{-n\left(m \lambda_{s}-\lambda\right)}(n, \varepsilon)$-balls. Since each "nice" $(n, \varepsilon)$-ball gives a contribution of measure at most $e^{-n h}$, a "nice" $\varepsilon e^{-n \lambda_{s}}$-ball should not contain measure more than $e^{-n\left(m \lambda_{s}-\lambda+h\right)}$.

Similarly, by comparing the volumes of an $\varepsilon e^{-n \lambda_{1}}$-ball and an $(n, \varepsilon)$-ball, we get that an $(n, \varepsilon)$-ball can be covered by $e^{n\left(m \lambda_{1}-\lambda\right)}$ balls of radius $\varepsilon e^{-n \lambda_{1}}$. Therefore, a general $\varepsilon e^{-n \lambda_{1}}$-ball should contribute measure at least $e^{-n\left(m \lambda_{1}-\lambda+h\right)}$.

The proof for the proposition carries out the above idea. However, since convergences in (2.5) and (3.1) are not uniform, we have to choose some "nice" sets on which the above argument can be applied. Also, we consider all $\varepsilon e^{-n \lambda_{s}}$-balls and $(n, \varepsilon)$-balls which cover the "nice" sets simultaneously.

Proposition 2. Let $0<\gamma<\frac{1}{10} \min \left\{\gamma_{0}, h\right\}$ and $0<\varepsilon<\frac{1}{10} \min \left\{\varepsilon_{1}, \varepsilon_{2}, \varepsilon_{3}, 1\right\}$. Then u-a.e. $x \in \Lambda$,

$$
\begin{gathered}
m-\frac{\lambda-h+(2 m+3) \gamma}{\lambda_{s}-2 \gamma} \leqq \liminf _{n \rightarrow \infty}-\frac{1}{n\left(\lambda_{s}-2 \gamma\right)} \log \mu B\left(x, \varepsilon e^{-n\left(\lambda_{s}-2 \gamma\right)}\right), \\
\limsup _{n \rightarrow \infty}-\frac{1}{n\left(\lambda_{1}+2 \gamma\right)} \log \mu B\left(x, \varepsilon e^{-n\left(\lambda_{1}+2 \gamma\right)}\right) \leqq m-\frac{\lambda-h-(2 m+3) \gamma}{\lambda_{1}+2 \gamma} .
\end{gathered}
$$

Proof of Proposition 2 (The First Inequality). Write $\lambda_{-}=\lambda_{s}-2 \gamma$.

Suppose this inequality is not true. Then by Lemma 3, for some small $\gamma$ and $\varepsilon$, $\mu$-a.e. $x \in \Lambda$,

$$
\liminf _{n \rightarrow \infty}-\frac{1}{n \lambda_{-}} \log \mu B\left(x, \varepsilon e^{-n \lambda_{-}}\right)<m-\frac{\lambda-h+(2 m+3) \gamma}{\lambda_{-}} .
$$

Fix $l>1$ such that $K l^{-1} \leqq 1$ and $\mu \Lambda_{l}>0$.

Put

$$
A_{n}^{\prime}=\left\{x \in \Lambda: \mu B_{k}\left(x, \varepsilon l^{-2} e^{-2 k \gamma}\right)<\exp [-k(h-\gamma)] \forall k \geqq n\right\}
$$


By Lemma $4 \lim _{n \rightarrow \infty} \mu A_{n}^{\prime}=1$, because $\mu B_{k}\left(x, \varepsilon l^{-2} e^{-2 k \gamma}\right) \leqq \mu B_{k}(x, \varepsilon) \forall k \geqq 0$.

Put

$$
A_{n}^{\prime \prime}=\left\{x \in \Lambda:\left|D f_{x}^{k}\right|<\exp k(\lambda+\gamma) \forall k \geqq n\right\} .
$$

By the fact that $\lambda=\lim _{n \rightarrow \infty} \frac{1}{n} \log \left|D f^{n}(x)\right| \mu$-a.e. $x \in \Lambda, \lim _{n \rightarrow \infty} \mu A_{n}^{\prime \prime}=1$.

Put

$$
A_{n}^{\prime \prime \prime}=\left\{x \in \Lambda: \mu B\left(x, \varepsilon e^{-n \lambda_{-}}\right) \geqq \exp \left[-n\left(m \lambda_{-}-\lambda+h-(2 m+3) \gamma\right)\right]\right\} .
$$

By (4.1) $\mu\left(\bigcup_{n=N}^{\infty} A_{n}^{\prime \prime \prime}\right)=1 \forall N>0$.

Put $A_{n}=A_{n}^{\prime} \cap A_{n}^{\prime \prime} \cap A_{n}^{\prime \prime \prime} \cap \Lambda_{l}$. Since both $\left\{A_{n}^{\prime}\right\}$ and $\left\{A_{n}^{\prime \prime}\right\}$ are increasing, we get $\mu\left(\bigcup_{n=N}^{\infty} A_{n}\right)=\mu \Lambda_{l}>0 \forall N>0$. So we can find an $n>0$ with $\mu A_{n}>8^{m} l^{2 m} K_{1} K_{2} e^{-n \gamma}$.

On the other hand, from the following three claims we get

$$
\begin{aligned}
\mu A_{n} & \leqq e^{n\left(m \lambda_{-}-\lambda+h-(2 m+3) \gamma\right)} \cdot 8^{m} l^{2 m} K_{1} K_{2} e^{n\left(-m \lambda_{-}+\lambda+(2 m+1) \gamma\right)} \cdot e^{-n(h-\gamma)} \\
& =8^{m} l^{2 m} K_{1} K_{2} e^{-n \gamma} .
\end{aligned}
$$

This contradiction means that (4.1) is not true.

Claim 1. The set $A_{n}$ can be covered by $e^{n\left(m \lambda_{-}-\lambda+h-(2 m+3) \gamma\right)}$ balls $B\left(x, 2 \varepsilon e^{-n \lambda_{-}}\right)$ with $x \in A_{n}$.

Claim 2. $\forall x \in A_{n}, B\left(x, 2 \varepsilon e^{-n \lambda_{-}}\right) \cap A_{n}$ can be covered by $8^{m} l^{2 m} K_{1} K_{2} e^{n\left(-m \lambda_{-}+\lambda+(2 m+1) \gamma\right)}$ $\left(n, \varepsilon l^{-2} e^{-2 n \gamma}\right)$-balls $B_{n}\left(y, \varepsilon l^{-2} e^{-2 n \gamma}\right)$ with $y \in A_{n}$.

Claim 3. $\forall y \in A_{n}, B_{n}\left(y, \varepsilon l^{-2} e^{-2 n \gamma}\right)$ has measure less than or equal to $e^{-n(h-\gamma)}$, i.e. $\mu B_{n}\left(y, \varepsilon l^{-2} e^{-2 n \gamma}\right) \leqq e^{-n(h-\gamma)}$.

Proof of Claim 1. Since $A_{n} \subset A_{n}^{\prime \prime \prime}$, each $\varepsilon e^{-n \lambda_{-}}$-ball about $x \in A_{n}$ has measure greater than or equal to $\exp \left[-n\left(m \lambda_{-}-\lambda+h-(2 m+3) \gamma\right)\right]$. So there are at most

$$
\exp n\left(m \lambda_{-}-\lambda+h-(2 m+3) \gamma\right)
$$

disjoint balls of radius $\varepsilon e^{-n \lambda_{-}}$about points in $A_{n}$. The same number of balls of radius $2 \varepsilon e^{-n \lambda_{-}}$about points in $A_{n}$ can cover $A_{n}$.

Proof of Claim 2. Since $A_{n} \subset \Lambda_{l}$, by Proposition 1 and the fact

$$
\frac{\varepsilon}{2} l^{-2} e^{-2 n \gamma} \cdot K l e^{-n\left(\lambda_{s}-3 \gamma\right)}=\frac{\varepsilon}{2} K l^{-1} e^{-n\left(\lambda_{s}-\gamma\right)}<\varepsilon e^{-n\left(\lambda_{s}-\gamma\right)}<\varepsilon e^{-n \lambda_{-}},
$$

we know that $B_{n}\left(y, \frac{\varepsilon}{2} l^{-2} e^{-2 n \gamma}\right) \subset B\left(y, \varepsilon e^{-n \lambda_{-}}\right) \forall y \in A_{n}$. So, if $y \in A_{n} \cap$ $B\left(x, 2 \varepsilon e^{-n \lambda_{-}}\right)$for some $x \in M$, then $B_{n}\left(y, \frac{\varepsilon}{2} l^{-2} e^{-2 n \gamma}\right) \subset B\left(x, 4 \varepsilon e^{-n \lambda_{-}}\right)$.

Since $A_{n} \subset A_{n}^{\prime \prime}$, Lemma 2 implies that for any $y \in A_{n}$,

$$
\begin{aligned}
v B_{n}\left(y, \frac{\varepsilon}{2} l^{-2} e^{-2 n \gamma}\right) & \geqq K_{2}^{-1}\left(\frac{\varepsilon}{2}\right)^{m} l^{-2 m} e^{-2 n m \gamma} \cdot e^{-n(\lambda+\gamma)} \\
& =\varepsilon^{m} 2^{-m} l^{-2 m} K_{2}^{-1} e^{-n(\lambda+(2 m+1) \gamma)}
\end{aligned}
$$

By Lemma 1,

$$
v B\left(x, 4 \varepsilon e^{-n \lambda_{-}}\right) \leqq 4^{m} K_{1} \varepsilon^{m} e^{-n m \lambda_{-}} .
$$


Hence, there are at most

$$
\varepsilon^{-m} 2^{m} l^{2 m} K_{2} e^{n(\lambda+(2 m+1) \gamma)} \cdot 4^{m} K_{1} \varepsilon^{m} e^{-n m \lambda_{-}}=8^{m} l^{2 m} K_{1} K_{2} e^{n\left(-m \lambda_{-}+\lambda+(2 m+1) \gamma\right)}
$$

disjoint $\left(n, \frac{\varepsilon}{2} l^{-2} e^{-2 n \gamma}\right)$-balls about points in $A_{n}$ contained in $B\left(x, 4 \varepsilon e^{-n \lambda_{-}}\right)$. So the same number of $\left(n, \varepsilon l^{-2} e^{-2 n \gamma}\right)$-balls can cover $B\left(x, 2 \varepsilon e^{-n \lambda_{-}}\right)$.

Proof of Claim 3. This is because $A_{n} \subset A_{n}^{\prime}$.

Proof of Proposition 2 (The Second Inequality). The proof is analogous to that for the first inequality. We only give an outline here.

Write $\lambda_{+}=\lambda_{1}+2 \gamma$. Suppose for some small $\gamma$ and $\varepsilon$,

$$
\limsup _{n \rightarrow \infty}-\frac{1}{n \lambda_{+}} \log \mu B\left(x, \varepsilon e^{-n \lambda_{+}}\right)>m-\frac{\lambda-h-(2 m+3) \gamma}{\lambda_{+}} \mu \text {-a.e. } x \in \Lambda \text {. }
$$

Fix $l \geqq 1$ such that $K l^{-1} \leqq 1$ and $\mu \Lambda_{l}>0$. Put

$$
\begin{aligned}
& A_{n}^{\prime}=\left\{x \in \Lambda: \mu B_{k}(x, \varepsilon)>\exp [-k(h+\gamma)] \forall k \geqq n\right\}, \\
& A_{n}^{\prime \prime}=\left\{x \in \Lambda:\left|D f_{x}^{k}\right|>\exp k(\lambda-\gamma) \forall k \geqq n\right\}, \\
& A_{n}^{\prime \prime \prime}=\left\{x \in \Lambda: \mu B\left(x, \varepsilon l^{-2} e^{-n\left(\lambda_{+}+2 \gamma\right)}\right) \leqq \exp \left[-n\left(m \lambda_{+}-\lambda+h+(2 m+3) \gamma\right)\right]\right\},
\end{aligned}
$$

and put $A_{n}=A_{n}^{\prime} \cap A_{n}^{\prime \prime} \cap A_{n}^{\prime \prime \prime} \cap \Lambda_{l}$. We can find an $n>0$ with $\mu A_{n}>8^{m} l^{2 m} K_{1} K_{2} e^{-n \gamma}$.

However, the following three claims give that

$$
\begin{aligned}
\mu A_{n} & \leqq e^{n(h+\gamma)} \cdot 8^{m} l^{2 m} K_{1} K_{2} e^{n\left(m \lambda_{+}-\lambda+(2 m+1) \gamma\right)} \cdot e^{-n\left(m \lambda_{+}-\lambda+h+(2 m+3) \gamma\right)} \\
& =8^{m} l^{2 m} K_{1} K_{2} e^{-n \gamma}
\end{aligned}
$$

a contradiction.

Claim 4. The set $A_{n}$ can be covered by $e^{n(h+\gamma)}(n, 2 \varepsilon)$-balls $B_{n}(x, 2 \varepsilon)$ centered at points $x \in A_{n}$.

Claim 5. $\forall x \in A_{n}, B_{n}(x, 2 \varepsilon)$ can be covered by $8^{m} l^{2 m} K_{1} K_{2} e^{n\left(m \lambda_{+}-\lambda+(2 m+1) \gamma\right)}$ balls $B\left(y, \varepsilon l^{-2} e^{-n\left(\lambda_{+}+2 \gamma\right)}\right)$ with center points $y \in A_{n}$.

Claim 6. $\forall y \in A_{n}$, the ball $B\left(y, \varepsilon l^{-2} e^{-n\left(\lambda_{+}+2 \gamma\right)}\right)$ has measure less than or equal to $e^{-n\left(m \hat{\lambda}_{+}-\lambda+h+(2 m+3) \gamma\right)}$.

Proof of Claim 4. Since $A_{n} \subset A_{n}^{\prime}$, there exist at most $e^{n(h+\gamma)}$ disjoint $(n, \varepsilon)$-balls $B_{n}(x, \varepsilon)$ with $x \in A_{n}$. Hence the same number of $(n, 2 \varepsilon)$-balls about points in $A_{n}$ can cover $A_{n}$.

Proof of Claim 5. Since $A_{n} \subset \Lambda_{l}$, by Proposition 1 we have $B\left(y, \frac{\varepsilon}{2} l^{-2} e^{-n\left(\lambda_{+}+2 \gamma\right)}\right)$ $\subset B_{n}\left(y, \varepsilon e^{-2 n \gamma}\right) \subset B_{n}(y, \varepsilon) \forall y \in A_{n}$. So if $y \in A_{n} \cap B_{n}(x, 2 \varepsilon)$ for some $x \in S$, then $B\left(y, \frac{\varepsilon}{2} l^{-2} e^{-n\left(\lambda_{+}+2 \gamma\right)}\right) \subset B_{n}(x, 4 \varepsilon)$.

Since $A_{n} \subset A_{n}^{\prime \prime}$, by Lemma 2 we get $v B_{n}(x, 4 \varepsilon) \leqq K_{2} 4^{m} \varepsilon^{m} e^{-n(\lambda-\gamma)} \forall x \in A_{n}$. By Lemma $1 v B\left(y, \frac{\varepsilon}{2} l^{-2} e^{-n\left(\lambda_{+}+2 \gamma\right)}\right) \geqq K_{1}^{-1} \varepsilon^{m} 2^{-m} l^{-2 m} e^{-n m\left(\lambda_{+}+2 \gamma\right)}$. So there are at most

$$
8^{m} l^{2 m} K_{1} K_{2} e^{n\left(m \lambda_{+}-\lambda+(2 m+1) \gamma\right)}
$$


disjoint balls of radius $\frac{\varepsilon}{2} l^{-2} e^{-n\left(\lambda_{+}+2 \gamma\right)}$ contained in $B_{n}(x, 4 \varepsilon)$. Therefore, $B_{n}(x$, $2 \varepsilon$ ) can be covered by the same number of $\varepsilon l^{-2} e^{-n\left(\lambda_{+}+2 \gamma\right)}$-balls.

Proof of Claim 6. This is because $A_{n} \subset A_{n}^{\prime \prime \prime}$.

To prove Theorem B, we also need the following fact, a version of which is in $[\mathrm{Y}]$.

Fact 2. Suppose $v$ is a Borel probability measure on a manifold $M$. If there is a sequence of positive real numbers $\left\{r_{n}\right\}$ with $\lim _{n \rightarrow \infty} r_{n}=0$ and $\lim _{n \rightarrow \infty} \frac{\log r_{n+1}}{\log r_{n}}=1$ such that

$$
\underline{\delta} \leqq \liminf _{n \rightarrow \infty} \frac{\log v B\left(x, r_{n}\right)}{\log r_{n}} \leqq \limsup _{n \rightarrow \infty} \frac{\log v B\left(x, r_{n}\right)}{\log r_{n}} \leqq \bar{\delta}
$$

for v-a.e. $x \in M$, then

$$
\underline{\delta} \leqq D(v) \leqq \bar{\delta}
$$

where $D(v)$ is $\operatorname{dim}_{H}(v), \underline{\operatorname{dim}}_{B}(v)$, or $\overline{\operatorname{dim}}_{B}(v)$.

It should be pointed out that by (2.2) and (2.4), $D(v)$ also can be $\operatorname{dim}_{L}(v)$ or $\overline{\operatorname{dim}}_{L}(v)$.

Proof of Theorem $B$.

i) Take $l>0$ such that $\mu \Lambda_{l}>0$. By Proposition 1, for $\mu$-a.e. $x \in \Lambda_{l}$, if $0<$ $\varepsilon \leqq \min \left\{\varepsilon_{3}, \frac{1}{2} l^{-2}\right\}$, then

$$
\begin{aligned}
\liminf _{n \rightarrow \infty} \frac{\log \mu B\left(x, \varepsilon e^{-n\left(\lambda_{1}+4 \gamma\right)}\right)}{\log \varepsilon e^{-n\left(\lambda_{1}+4 \gamma\right)}} & \geqq \liminf _{n \rightarrow \infty}-\frac{1}{n\left(\lambda_{1}+4 \gamma\right)} \log \mu B_{n}\left(x, \varepsilon K l e^{-2 n \gamma}\right) \\
& \geqq \liminf _{n \rightarrow \infty}-\frac{1}{n\left(\lambda_{1}+4 \gamma\right)} \log \mu B_{n}(x, \varepsilon)=\frac{h}{\lambda_{1}+4 \gamma} .
\end{aligned}
$$

Since $\gamma$ is arbitrary, by Fact 2 we have $D(\mu) \geqq \frac{h}{\lambda_{1}}$. The upper bound $D(\mu) \leqq \frac{h}{\lambda_{s}}$ can be obtained similarly.

ii) This follows from Proposition 2 and Fact 2.

\section{Proof of Theorem A}

Take $r>0$ such that for some $1<\kappa^{\prime} \leqq \kappa, \quad\|D f(y) u\| \geqq \kappa^{\prime}\|u\| \forall y \in B(\Lambda, r)$ $\forall u \in T_{y} M$. This is possible because $f$ is uniformly expanding on $\Lambda$.

Proposition 3. For any $\varepsilon<\min \left\{r, \frac{1}{2} \varepsilon_{2}\right\}$,

$$
P(f,-\log |D f(x)|)=\lim _{n \rightarrow \infty} \frac{1}{n} \log v B_{n}(\Lambda, \varepsilon) \leqq 0 .
$$

Proof. Denote $\phi(x)=-\log |D f(x)|$ and $S_{n} \phi(x)=\sum_{i=0}^{n} \phi\left(f^{i} x\right)=-\log \left|D f^{n}(x)\right|$.

Take $0<\eta<\varepsilon$. Since $f$ is expanding, there exists $k>0$ such that if $x \in \Lambda, y \in B(\Lambda, r)$ satisfy $d\left(f^{i} x, f^{i} y\right) \leqq \varepsilon \forall 0 \leqq i \leqq k$, then $d(x, y) \leqq \eta$. So $B_{n+k}$ $(\Lambda, \varepsilon) \subset B_{n}(\Lambda, \eta) \forall n \geqq 0$. 
Take an $(n, \eta)$ spanning set $S$ for $\Lambda$. We have

$$
B_{n+k}(\Lambda, \varepsilon) \subset B_{n}(\Lambda, \eta) \subset \bigcup_{x \in S} B_{n}(x, 2 \eta) .
$$

By Lemma 2, $v B_{n}(x, 2 \eta) \leqq K_{2} 2^{m} \eta^{m}\left|D f^{n}(x)\right|^{-1}=K_{2} 2^{m} \eta^{m} e^{S_{n} \phi(x)}$. Thus,

$$
v B_{n+k}(\Lambda, \varepsilon) \leqq \sum_{x \in S} v B_{n}(x, 2 \eta) \leqq K_{2} 2^{m} \eta^{m} \sum_{x \in S} e^{S_{n} \phi(x)} .
$$

So, with the same notation as in [W] Sect. 9.1, we get

$$
\begin{aligned}
v B_{n+k}(\Lambda, \varepsilon) & \leqq K_{2} 2^{m} \eta^{m} Q_{n}(f, \phi, \eta), \\
\limsup _{n \rightarrow \infty} \frac{1}{n} \log v B_{n}(\Lambda, \varepsilon) & \leqq \limsup _{n \rightarrow \infty} \frac{1}{n} \log Q_{n}(f, \phi, \eta) .
\end{aligned}
$$

Since the left-hand side is independent of $\eta$, we have

$$
\limsup _{n \rightarrow \infty} \frac{1}{n} \log v B_{n}(\Lambda, \varepsilon) \leqq P(f, \phi) .
$$

The opposite inequality can be proved in an analogous way.

Before proving Theorem A, we give the following estimates.

Proposition 4. With the notations as in Theorem A,

i) $\frac{h_{\text {top }}}{\log \left\|\left.D f\right|_{\Lambda}\right\|} \leqq D(\Lambda) \leqq \frac{h_{\text {top }}}{\left.\log \llbracket D f\right|_{\Lambda} \rrbracket}$, and

ii) $m+\frac{P}{\left.\log \llbracket D f\right|_{\Lambda} \rrbracket} \leqq D(\Lambda) \leqq m+\frac{P}{\log \left\|\left.D f\right|_{\Lambda}\right\|}$.

Proof. Note that for any invariant measure $\mu$ on $\Lambda, \log \left\|\left.D f\right|_{\Lambda}\right\| \geqq \lambda_{1}$ and $\log \|\left. D f\right|_{\Lambda} \rrbracket$ $\leqq \lambda_{s}$. Therefore the lower bound $D(\Lambda) \geqq \frac{h_{\text {top }}}{\log \left\|\left.D f\right|_{\Lambda}\right\|}$ in Part i) follows from (2.1), (2.2), Theorem B and the fact that $h_{\text {top }}=\sup \left\{h_{\mu}(f): \mu \in \mathscr{E}(\Lambda, f)\right\}$, where $\mathscr{E}(\Lambda, f)$ is the set of all ergodic measures of $f$ on $\Lambda$.

By using the facts that $P=\sup \left\{h_{\mu}(f)-\int \log |D f(x)| d \mu(x): \mu \in \mathscr{E}(\Lambda, f)\right\}$ and $\lambda=\int \log |D f(x)| d \mu(x)$, we can obtain the lower bound $D(\Lambda) \geqq m+\frac{P}{\left.\log \llbracket D f\right|_{\Lambda} \rrbracket}$. that

Now we prove the upper bounds in both parts. By (2.3) we only need prove

$$
\overline{\operatorname{dim}}_{B}(\Lambda) \leqq \min \left\{\frac{h_{\text {top }}}{\log \left\|\left.D f\right|_{\Lambda}\right\|}, m+\frac{P}{\log \left\|\left.D f\right|_{\Lambda}\right\|}\right\} .
$$

For any $0<\beta<\left.\llbracket D f\right|_{\Lambda} \rrbracket-1$, there exists $\varepsilon_{0}>0$ such that $\forall y \in B\left(\Lambda, \varepsilon_{0}\right)$, $\|D f(y)\| \geqq \|\left. D f\right|_{\Lambda} \rrbracket-\beta$ and $\|D f(y)\| \leqq\left\|\left.D f\right|_{\Lambda}\right\|+\beta$. Denote

$$
\chi_{-}=\log \left(\left.\llbracket D f\right|_{\Lambda} \rrbracket-\beta\right) \text { and } \chi_{+}=\log \left(\left\|\left.D f\right|_{\Lambda}\right\|+\beta\right) .
$$

We assume $0<\varepsilon<\min \left\{r, \varepsilon_{1}, \frac{1}{2} \varepsilon_{2}, \frac{1}{2} \varepsilon_{0}\right\}$ so that Lemma 1 and Proposition 3 can be applied to this $\varepsilon$ as well.

We have that $\forall x \in B(\lambda, \varepsilon), B_{n}(x, \varepsilon) \subset B\left(x, \varepsilon\left(\left.\llbracket D f\right|_{\Lambda} \rrbracket-\beta\right)^{-n}\right)=B\left(x, \varepsilon e^{-n \chi_{-}}\right)$. So, if $S$ is an $(n, \varepsilon)$ spanning set for $\Lambda$, then $\left\{B\left(x, \varepsilon e^{-n \chi_{-}}\right): x \in S\right\}$ is a cover of $\Lambda$. This implies that

$$
N\left(\varepsilon e^{-n \chi_{-}}\right) \leqq N(n, \varepsilon) \text {, }
$$


where $N(n, \varepsilon)$ is the smallest cardinality of any $(n, \varepsilon)$ spanning set for $\Lambda$, and $N\left(\varepsilon e^{-n \chi_{-}}\right)$is the minimum number of balls of radius $\varepsilon e^{-n \chi_{-}}$which cover $\Lambda$. By the definitions of $\overline{\operatorname{dim}}_{B}(\Lambda)$ and topological entropy,

$$
\overline{\operatorname{dim}}_{B}(\Lambda)=\limsup _{n \rightarrow 0} \frac{\log N\left(\varepsilon e^{-n \chi_{-}}\right)}{-\log \varepsilon e^{-n \chi_{-}}} \leqq \limsup _{n \rightarrow 0} \frac{\log N(n, \varepsilon)}{n \chi_{-}}=\frac{h_{\text {top }}}{\chi_{-}} .
$$

By Proposition 3, there exists $N>0$ such that if $n>N$, then $v B_{n}(\Lambda, \varepsilon)<$ $e^{n(P+\beta)}$. Any $\varepsilon e^{-n \chi+}$-ball about a point in $\Lambda$ must be contained in $B_{n}(\Lambda, \varepsilon)$. Every such $\varepsilon e^{-n \chi+}$-ball has volume larger than $K_{1}^{-1} \varepsilon^{m} e^{-n m \chi+}$. So $B_{n}(\Lambda, \varepsilon)$ contains at most $K_{1} \varepsilon^{-m} e^{n\left(m \chi_{+}+P+\beta\right)}$ disjoint $\varepsilon e^{-n \chi_{+}}$-balls centered in $\Lambda$. This implies that

$$
N\left(2 \varepsilon e^{-n \chi_{+}}\right) \leqq K_{1} \varepsilon^{-m} e^{n\left(m \chi_{+}+P+\beta\right)} .
$$

Thus

$$
\overline{\operatorname{dim}}_{B}(\Lambda) \leqq \limsup _{n \rightarrow \infty} \frac{\log N\left(2 \varepsilon e^{-n \chi_{+}}\right)}{-\log 2 \varepsilon e^{-n \chi_{+}}} \leqq m+\frac{P+\beta}{\chi_{+}} .
$$

Since $\beta$ is arbitrary, (5.1) follows from (5.2)-(5.4). This finishes the proof.

Proof of Theorem A. Since $h\left(\left.f^{n}\right|_{\Lambda}\right)=n h_{\text {top }}$ and $P\left(\left.f^{n}\right|_{\Lambda},-\log \left|D f^{n}(x)\right|\right)=n P$, we can use $f^{n}$ instead of $f$ and then take limit to obtain Theorem A from Proposition 4.

Acknowledgement. I would like to thank Professor Lai-Sang Young, my former advisor, for many important suggestions. I would also thank the referee for carefully reading the manuscript and for suggesting various improvements.

\section{References}

[B] Bedford, T.: Crinkly curves, Markov partitions and box dimension in self-similar sets. Ph.D. Thesis, University of Warwick

[BK] Brin, M., Katok, A.: On local entropy. In: Geometric dynamics. Lecture notes in mathematics No. 1007, Berlin, Heidelberg, New York: Springer, 1983, pp. 30-38

[BU] Bedford, T., Urbański, M.: The box and Hausdorff dimension of self-affine sets. Ergodic Theory and Dynamical Systems. 10, 627-644 (1990)

[F1] Falconer, K.: The Hausdorff dimension of self-affine fractals. Math. Proc. Camb. Phil. Soc. 103, 339-350 (1988)

[F2] Falconer, K.: Fractal geometry-mathematical foundations and applications. New York: Wiley, 1990

[G] Gu, X.: An upper bound for the Hausdorff dimension of a hyperbolic set. Nonlinearity 4, 927-934 (1991)

[GL] Gatzouras, D., Lalley, S.: Hausdorff and box dimensions of certain self-affine fractals. Indiana Univ. Math. J. 41, 533-568 (1992)

[H] Hutchinson, J.E.: Fractals and self similarity. Indiana Univ. Math. J. 30, 713-747 (1981)

[L] Ledrappier, F.: Some relations between dimension and Lyapunov exponents. Commun. Math. Phys. 81, 229-238 (1981)

[LY] Ledrappier, F., Young, L-S.: The metric entropy of diffeomorphisms. Ann. Math. 122, 509-574 (1985)

[Mc] McMullen, C.: The Hausdorff dimension of general Sierpinski carpets. Nagoya Math. J. 96, 1-9 (1984)

[MM] McCluskey, H., Manning, A.: Hausdorff dimension for horseshoe. Ergodic Theory and Dynamical Systems 3, 251-260 (1983) 
[P] Petersen, K.: Ergodic theory. Cambridge: Cambridge University Press, 1983

[R] Ruelle, D.: Bowen's formula for the Hausdorff dimension of self-similar sets. In: Scaling and self-similarity in physics, Progress in Physics 7. Frohlich, J. (ed.). Boston: Birkhäuser, 1983

[W] Walters, P.: An introduction to ergodic theory. Graduate Texts in Mathematics, vol. 79. Berlin, Heidelberg, New York: Springer, 1981

[Y] Young, L.-S.: Dimension, entropy and Lyapunov exponents. Ergodic Theory and Dynamical Systems 2, 109-129 (1982)

Communicated by J.-P. Eckmann 